\title{
An Unusual Solitary Prostate Cancer Metastasis Detected by Gallium-68 Prostate-specific Membrane Antigen- labeled Positron Emission Tomography/Magnetic Resonance Imaging
}

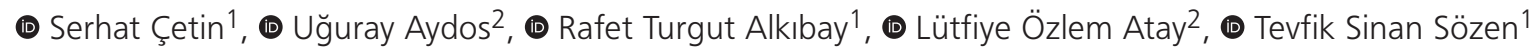 \\ ${ }^{1}$ Gazi University Faculty of Medicine, Department of Urology, Ankara, Turkey \\ ${ }^{2}$ Gazi University Faculty of Medicine, Department of Nuclear Medicine, Ankara, Turkey
}

\begin{abstract}
The two most common sites of prostate cancer metastasis include the lymph nodes and bone. Solid-organ metastases usually occur in the lungs, liver, and brain. Prostate adenocarcinoma metastasis to the skin and subcutaneous cellular tissue occurs in $<0.3 \%$ and are thus considered exceptional. Herein, we report a unique case of a pectoral subcutaneous metastasis as the first recurrence site after definitive local and systemic therapy for prostate cancer, which was identified by gallium-68 prostate-specific membrane antigen-labeled positron emission tomography/magnetic resonance imaging.
\end{abstract}

Keywords: Prostate cancer, metastasis, unusual, Ga-68 PSMA PET/MRI

\section{Introduction}

Prostate cancer is one of the most important health problems of males today. According to the 2018 GLOBOCAN data, prostate cancer is the second most common cancer in males but is the eighth in cancer-related deaths (1). The most frequent histologic type is adenocarcinoma. The two most common sites of metastasis include the lymph nodes and bone. Solid-organ metastases usually occur in the lungs, liver, and brain (2). Prostate adenocarcinoma metastasis to the skin and subcutaneous cellular tissue occurs in $<0.3 \%$ and is thus considered exceptional (3). Herein, we report a unique case of a pectoral subcutaneous metastasis as the first recurrence site after definitive local and systemic therapy for prostate cancer, which was identified by Gallium-68 (Ga-68) prostatespecific membrane antigen (PSMA)-labeled positron emission tomography/magnetic resonance imaging (PET/MRI).

\section{Case Report}

A 66-year-old male patient had an International Society of Urological Pathology Grade 5 prostate adenocarcinoma with a prostate-specific antigen (PSA) level of $7 \mathrm{ng} / \mathrm{mL}$. Ga-68 PSMA PET/MRI examination at the time of diagnosis did not reveal any systemic metastasis but showed right seminal vesicle invasion. The patient received neoadjuvant chemotherapy (six cycles of docetaxel) along with androgen ablation (after the third cycle of chemotherapy). Then, robot-assisted laparoscopic radical prostatectomy and lymph node dissection were performed. The pathological stage was pT3b N0. Surgical margins were negative, but the seminal vesicle invasion was confirmed. Androgen ablation was postoperatively stopped. Radiotherapy to prostatic bed and pelvis was planned at 6 months after continence was sufficiently achieved. Postoperative nadir PSA was $0 \mathrm{ng} / \mathrm{mL}$ at 1 month, which did not change during the follow-up. On postoperative 18 months, PSA recurred as 0.1 and $0.2 \mathrm{ng} / \mathrm{mL}$ in two consecutive tests, and immediately, a new Ga-68 PSMA-labeled PET/MRI examination was done. Only a $15 \times 9 \mathrm{~mm}$ subcutaneous soft tissue nodule on the right pectoral region with increased Ga-68 PSMA uptake (SUV max $_{\text {: }}$ 7.5) was detected on maximum intensity projection (MIP) image, axial T1-w, and fusion PET/MRI images (red arrows, Figure 1a, $1 \mathrm{~b}$, and 1c). Axial diffusion-weighted image (DWl, b: 1000) and apparent diffusion coefficient (ADC) map, which was performed

Cite this article as: Çetin S, Aydos U, Alkıbay RT, Atay LÖ, Sözen TS. An Unusual Solitary Prostate Cancer Metastasis Detected by Gallium-68 Prostate-specific Membrane Antigen-labeled Positron Emission Tomography/Magnetic Resonance Imaging. Bull Urooncol 2022;21(1):32-34 
a

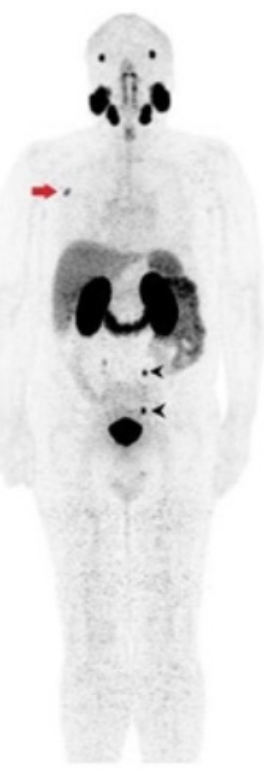

b

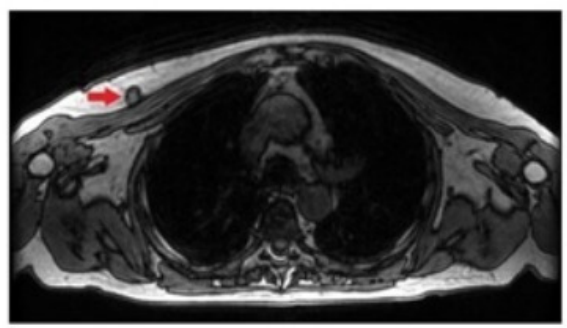

c

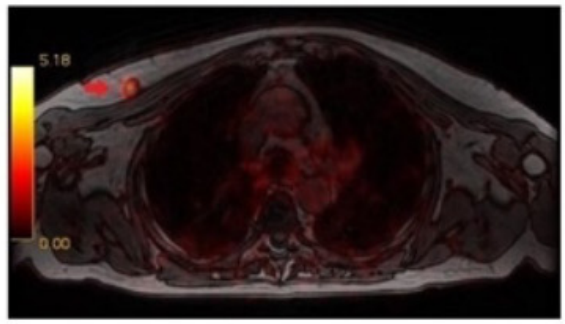

d

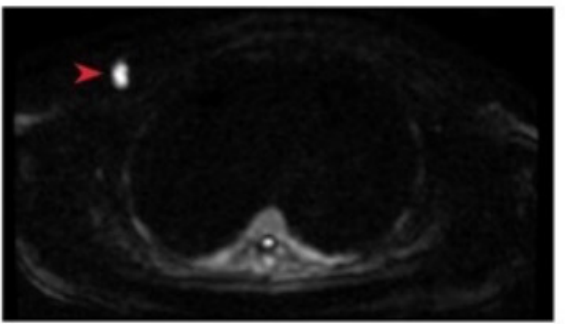

e

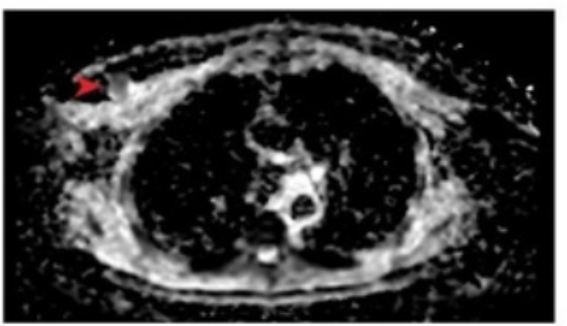

Figure 1a,b,c,d,e. Ga-68 PSMA PET/MRI image

PSMA: Prostate-specific membrane antigen, PET: Positron emission tomography, MRI: Magnetic resonance imaging

in the same imaging session with PET/MRI, also revealed increased signal and diffusion restriction on this lesion (red arrowheads, Figure $1 \mathrm{~d}$ and $1 \mathrm{e}$ ). On the MIP image, urinary activities were also seen in the left ureter (black arrowheads, Figure 1a). The nodule was excised, and the pathological examination revealed a wellcapsulated adenocarcinoma metastasis with negative surgical margins in the subcutaneous fat tissue. Since then, the patient was followed with a $0-n g / m L$ PSA level and without disease evidence. This article was written by taking informed consent form from the patient.

\section{Discussion}

PSMA ligand PET/computed tomography (CT) or PET/ MRI has high sensitivity and specificity for prostate cancer staging (4). Furthermore, Ga-68-labeled PSMA PET imaging demonstrated a higher detection rate of $45 \%$ in patients with biochemical recurrence (BR) and PSA values of $0.2-0.49 \mathrm{ng} /$ $\mathrm{mL}$ compared with the other conventional imaging methods (5). Previous studies $(6,7,8)$ reported $47 \%$ and $57 \%$ detection rates for patients with PSA values of $\leq 0.2 \mathrm{ng} / \mathrm{mL}$ and $0.2-0.5$ $\mathrm{ng} / \mathrm{mL}$ after radical prostatectomy. PET/MRI showed higher detection rates in patients with BR compared to the PET/CT due to the additional diagnostic value of MRI with superior soft tissue contrast. A recent study (9) revealed that Ga-68 PSMA PET/MRI showed PSMA-positive lesions in $65 \%$ of patients with a PSA level of 0.2-0.5 ng/mL. Recurrent lesions are mostly located in the prostatic bed, lymph nodes, and bones.

Ga-68 PSMA PET/MRI was performed in our reported case with BR after radical prostatectomy. Only a subcutaneous soft tissue nodule on the right pectoral region with increased Ga68 PSMA uptake on PET, increased signal intensity on DWI, and diffused restriction on ADC map was detected. According to the proposed structured reporting system for PSMA PET imaging,
PSMA-RADS Version 1.0 (10), the lesion detected on PSMA PET/ MRI was considered as PSMA-RADS-3C because of the intense uptake but highly atypical localization for prostate cancer. Additionally, excisional biopsy to confirm diagnosis histologically was performed, and pathological examination revealed prostatic adenocarcinoma metastasis. The subcutaneous dissemination of prostate cancer is a rare and unusual metastatic site. Previous case reports have reported patients with skin and subcutaneous metastases from small cell carcinoma of the prostate $(11,12)$, but our case did not have neuroendocrine differentiation. Subcutaneous metastasis may be seen in patients in advanced stages and terminal phases (13), but unusual as the first and only site.

Considering its higher sensitivity and specificity, whole-body Ga-68 PSMA-labeled PET/MRI contributes to the diagnostic work-up and restaging of patients with BR during prostatic bed evaluation, as well as in atypically located metastatic lesion detection.

\section{Acknowledgements}

Publication: The results of the study were not published in full or in part in form of abstracts.

Contribution: There is not any contributors who may not be listed as authors.

Conflict of Interest: No conflict of interest was declared by the authors.

Financial Disclosure: The authors declared that this study received no financial support.

Ethics

Informed Consent: This article was written by taking informed consent form from the patient.

Peer-review: Externally peer-reviewed. 


\section{Authorship Contributions}

Supervision: L.Ö.A., Concept: T.S.S., Design: R.T.A., Data Collection or Processing: U.A., S.Ç., Analysis or Interpretation: U.A., S.Ç., Literature Search: U.A., Writing: S.Ç.

\section{References}

1. Bray F, Ferlay J, Soerjomataram I, et al. Global cancer statistics 2018: GLOBOCAN estimates of incidence and mortality worldwide for 36 cancers in 185 countries. CA Cancer J Clin 2018;68:394-424.

2. Alonso AR, Freire FD, Garcia DP, et al. Metástasis de adenocarcinoma prostático en saco herniario. Actas Urol Esp 1999;23:717-719.

3. Gallego Sánchez JA, Astobieta Odriozola A, Alvarez Martínez J, et al. Skin metastasis as first manifestation of prostatic adenocarcinoma. Actas Urol Esp 1998;22:770-772.

4. Perera M, Papa N, Christidis D, et al. Sensitivity, specificity, and predictors of positive $68 \mathrm{Ga}$-prostate-specific membrane antigen positron emission tomography in advanced prostate cancer: a systematic review and meta-analysis. Eur Urol 2016;70:926-937.

5. Perera M, Papa N, Roberts $M$, et al. Gallium-68 Prostate-specific Membrane Antigen Positron Emission Tomography in Advanced Prostate Cancer-Updated Diagnostic Utility, Sensitivity, Specificity, and Distribution of Prostate-specific Membrane Antigen-avid Lesions: A Systematic Review and Meta-analysis. Eur Urol 2020;77:403-417.

6. Afshar-Oromieh A, Avtzi E, Giesel FL, et al. The diagnostic value of $\mathrm{PET} / \mathrm{CT}$ imaging with the (68)Ga-labelled PSMA ligand HBED-CC in the diagnosis of recurrent prostate cancer. Eur J Nucl Med Mol Imaging 2015;42:197-209.

7. Afshar-Oromieh $\mathrm{A}$, Holland-Letz $\mathrm{T}$, Giesel $\mathrm{FL}$, et al. Diagnostic performance of 68Ga-PSMA-11 (HBED-CC) PET/CT in patients with recurrent prostate cancer: evaluation in 1007 patients. Eur J Nucl Med Mol Imaging 2017;44:1258-1268.

8. Eiber M, Maurer T, Souvatzoglou M, et al. Evaluation of hybrid $68 \mathrm{Ga}$ PSMA ligand PET/CT in 248 patients with biochemical recurrence after radical prostatectomy. J Nucl Med 2015;56:668-674.

9. Kranzbühler B, Müller J, Becker AS, et al. Detection rate and localization of prostate cancer recurrence using 68Ga-PSMA-11 PET/MRI in patients with low PSA values $\leq 0.5 \mathrm{ng} / \mathrm{ml}$. J Nucl Med 2020;61:194-201.

10. Rowe SP, Pienta KJ, Pomper MG, Gorin MA. Proposal for a structured reporting system for prostate-specific membrane antigen-targeted PET imaging: PSMA-RADS version 1.0. J Nucl Med 2018;59:479-485.

11. Kaplan M, Atakan IH, Bilgi S, Inci O. Case report: subcutaneous metastasis from small cell carcinoma of the prostate. Int Urol Nephrol 2007;39:157-160.

12. Cecen K, Karadag MA, Demir A, Kocaaslan R. Small cell carcinoma of the prostate presenting with skin metastasis: a case report. J Med Case Rep 2014;8:146.

13. Herrera Puerto J, Pierna Manzano J, Goméz Tejeda LM, Isusquiza Garro I. Subcutaneous supramammary metastasis of prostatic carcinoma. Actas Urol Esp 1999;23:367-369. 